\title{
Infarto Agudo do Miocárdio de Parede Inferior sem Lesão Obstrutiva Coronária
}

\author{
Ricardo Wang ${ }^{1,2}$, Newton Stadler Souza Filho ${ }^{1,3}$, José Augusto Ribas Fortes ${ }^{1,3}$, Lidia Zytinski Moura ${ }^{1,2}$, \\ Andre Bernardi ${ }^{1}$, Leonardo Spolador ${ }^{1}$, Elide Sbardellotto Mariano da Costa ${ }^{1}$, \\ Renata Fortes Etchepare', José Rocha Faria Neto ${ }^{1,2}$
}

\section{RESUMO}

Descrevemos um caso de paciente com 70 anos, admitida com quadro compatível de infarto agudo do miocárdio, com sinais clínicos compatíveis, bem como alterações eletrocardiográficas, enzimáticas e de contratilidade. À angiografia coronária não foram evidenciadas lesões obstrutivas, o que foi confirmado pela avaliação com ultrassonografia intracoronária. Os autores discutem as hipóteses levantadas para explicar o infarto do miocárdio com artérias coronárias normais.

DESCRITORES: Infarto do miocárdio. Doença das coronárias. Angiografia coronária. Ultra-sonografia de intervenção.

0 infarto agudo do miocárdio é a principal causa de morbidade e mortalidade em todo o mundo'. A maioria dos casos decorre da instabilização de uma placa aterosclerótica com trombose sobrejacente. Vários outros mecanismos foram identificados como causa de síndrome coronária aguda, como obstrução dinâmica, inflamação, obstrução mecânica e aumento de consumo miocárdico de oxigênio. Descrevemos a seguir um caso de infarto agudo do miocárdio com coronárias normais.

\section{RELATO DO CASO}

Paciente E.K.S., sexo feminino, 70 anos, leucoderma, residente em Curitiba (PR), aposentada. Relata que, em 12/12/2008, por volta das 21 horas, apresentou quadro de dor torácica retroesternal em aperto, de

\footnotetext{
1 Santa Casa de Curitiba - Curitiba, PR, Brasil.

2 Pontifícia Universidade Católica do Paraná - Curitiba, PR, Brasil.

3 Instituto de Neurologia e Cardiologia de Curitiba - Curitiba, PR, Brasil.

Correspondência: Ricardo Wang. Rua Morretes, 252/504 - Portão Curitiba, PR, Brasil - CEP 80610-150

E-mail: rwang@terra.com.br

Recebido em: 8/1/2009 • Aceito em: 7/8/2009
}

\begin{abstract}
Inferior Acute Myocardial Infarction without Coronary Lesions

We describe the case of a 70-year-old patient admitted with acute myocardial infarction, with clinical, electrocardiographic, enzymatic and left ventricular regional wall motion abnormalities. Coronary angiography showed no obstructive lesions, which was confirmed by intravascular ultrasound (IVUS). Different hypothesis are raised to explain myocardial infarction with normal coronary arteries in this patient.
\end{abstract}

DESCRIPTORS: Myocardial infarction. Coronary disease. Coronary angiography. Ultrasonography, interventional.

início súbito e de grande intensidade, com irradiação para o membro superior direito, e duração aproximada de 30 minutos. A dor ocorreu em repouso sem fator desencadeante, e a paciente negou estresse emocional precedente à dor. Além disso, apresentou palidez cutânea, negando dispneia, palpitação, náuseas ou vômitos. Na avaliação dos fatores de risco cardiovascular, relatou ter diabetes melito controlado em uso de glibenclamida e metformina, hipertensão arterial tratada com captopril, negou tabagismo, e fazia uso crônico de ácido acetilsalicílico. Negou história recente compatível com quadro infeccioso.

Procurou, com esse quadro, assistência médica em uma unidade de emergência 24 horas, sendo realizado eletrocardiograma por volta das $21 \mathrm{~h} 30$, que demonstrou corrente de lesão subepicárdica em DII, DIII e AVF (Figura 1A). O eletrocardiograma sequencial, realizado duas horas após a admissão, demonstrou redução da onda $\mathrm{R}$ em DIII e AVF (Figura 1B). Na ocasião, foi administrada dose de ataque de ácido acetilsalicílico, heparinização plena e nitroglicerina. Com essas medidas, houve regressão da dor e do supradesnivelamento do segmento ST, porém a paciente evoluiu com área de necrose em parede inferior (Figura 1C). 


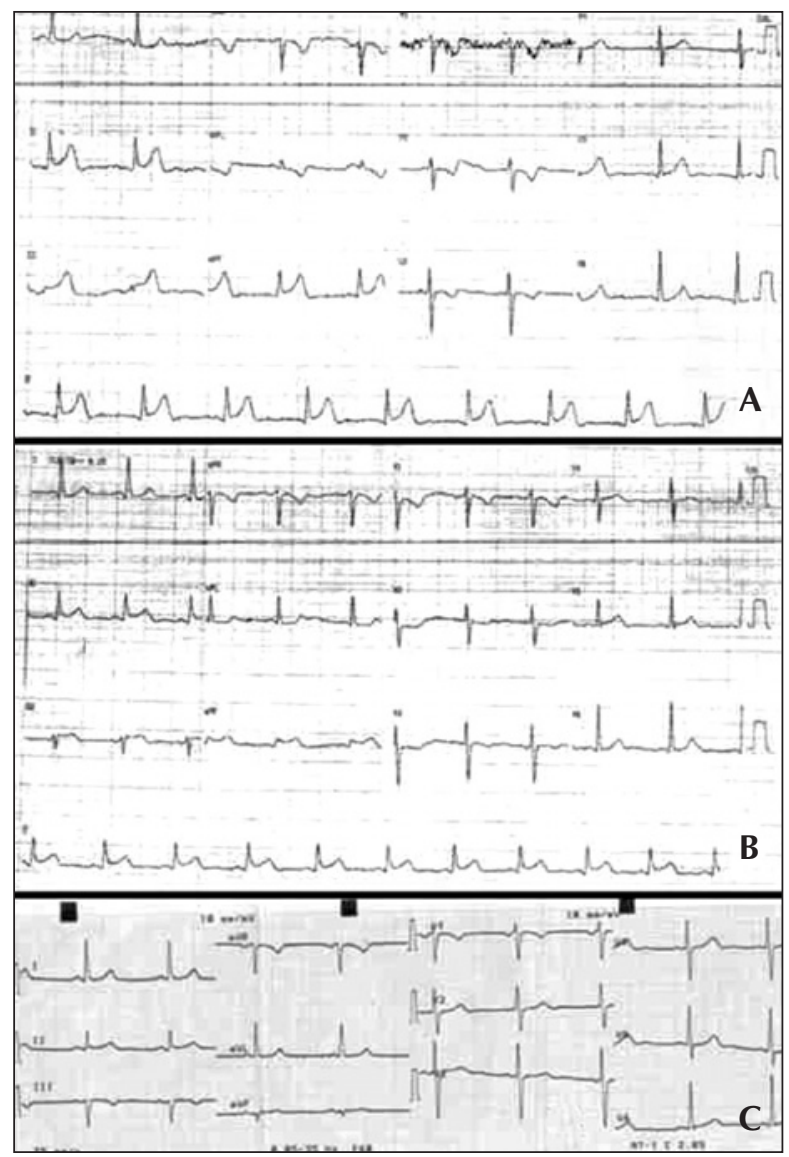

Figura 1 - Em A, eletrocardiograma à admissão na unidade de emergência 24 horas, demonstrando supradesnivelamento do segmento ST em DII, DIII e AVF. Em B, eletrocardiograma realizado duas horas após admissão na unidade de emergência 24 horas, demonstrando redução da onda R em DIII e AVF. Em C, eletrocardiograma à admissão na Santa Casa de Curitiba, demonstrando normalização do segmento ST com área de necrose inferior.

A paciente foi encaminhada para a Santa Casa de Curitiba em 13/12/2008, 12 horas após o início do quadro. À admissão, encontrava-se estável hemodinamicamente, com pressão arterial de 150/80 mmHg, frequência cardíaca de 56 bpm e respiratória, de 15 irpm. Apresentava obesidade principalmente abdominal, com índice de massa corporal de $33,2 \mathrm{~kg} / \mathrm{cm}^{2}$ e cintura abdominal de $116 \mathrm{~cm}$. O exame laboratorial demonstrava alterações enzimáticas compatíveis com infarto agudo do miocárdio, sendo a troponina T de $68 \mathrm{ng} / \mathrm{l}$ e a creatina quinase fração MB $(\mathrm{CK}-\mathrm{MB})$ de $100 \mathrm{U} / \mathrm{I}(\mathrm{VR}<16)$.

A paciente foi estratificada como de alto risco, em decorrência das alterações eletrocardiográficas e enzimáticas, optando-se por realização de estudo hemodinâmico. À ventriculografia, notava-se presença de hipocinesia de parede inferior, com função ventricular sistólica global preservada e fração de ejeção do ventrículo esquerdo estimada de 61\% (Figura 2A). Na artéria coronária direita dominante observava-se ausência de lesões obstrutivas e fluxo lento (Figura 2B), padrão similar ao encontrado na artéria coronária esquerda (Figura 2C).

Em vista das alterações eletrocardiográficas, enzimáticas e à ventriculografia, optou-se por realizar estudo com ultrassonografia intracoronária. A artéria coronária direita e seu ramo ventricular posterior direito não apresentavam doença aterosclerótica ou espessamento da íntima (> $5 \mathrm{~mm}$ ), como pode ser observado na Figura 2.

A paciente foi submetida a tratamento médico com dupla antiagregação plaquetária (ácido acetilsalicílico e clopidogrel), estatina e inibidor da enzima conversora da angiotensina, evoluindo com melhora clínica e recebendo alta hospitalar no sétimo dia.

\section{DISCUSSÃO}

Em 2007, as Sociedades Americana, Europeia e Mundial de Cardiologia se reuniram e unificaram os critérios para o diagnóstico de infarto agudo do miocárdio, com base nessa nova definição universal. O caso apresentado preenche os critérios para seu diagnóstico, pois conta com elevação enzimática associada a sinais clínicos, alterações eletrocardiográficas e de contratilidade segmentar ${ }^{1}$. A evolução eletrocardiográfica é típica de infarto agudo do miocárdio com supradesnivelamento do segmento $\mathrm{ST}^{2}$, inicialmente com lesão miocárdica aguda, que evoluiu para formação de necrose em parede inferior (onda Q). A rápida normalização do segmento ST indica a possibilidade de reperfusão coronária ${ }^{3}$. A elevação transitória classifica o paciente como de alto risco e, além do tratamento clínico intensivo, a estratégia invasiva precoce se faz necessária ${ }^{4}$.

A maioria dos casos de infarto agudo do miocárdio é secundária ao processo aterosclerótico, com ruptura e consequente trombose sobre placa ${ }^{5}$; somente $1 \%$ a $12 \%$ dos pacientes apresentam artérias coronárias com irregularidades à angiografia ${ }^{5-7}$. Essa incidência também foi observada por Waller ${ }^{6}$, na necropsia de pacientes que faleceram de infarto agudo do miocárdio, em que 95\% dos casos apresentaram instabilização da placa de ateroma. Em decorrência do remodelamento vascular promovido pela placa, pode haver perda de sensibilidade diagnóstica da angiografia na detecção de lesões com remodelamento positivo ${ }^{5,7}$. Baseados nessa premissa, vários autores ${ }^{5-7}$ defendem o uso rotineiro de ultrassonografia intracoronária em pacientes com essas características. Utilizando-se esse método, a real incidência de infarto agudo do miocárdio com artérias coronárias normais poderia estar abaixo de $1 \%{ }^{5}$; além disso, a ultrassonografia intracoronária permite o diagnóstico de outras afecções ${ }^{5}$. Esse fato poderia explicar a sobrevida observada no trabalho de Larsen et al. ${ }^{8}$, no qual os pacientes com artérias coronárias normais comparados ao grupo que apresentava lesão $<50 \%$ não tinham diferença na mortalidade, ainda 

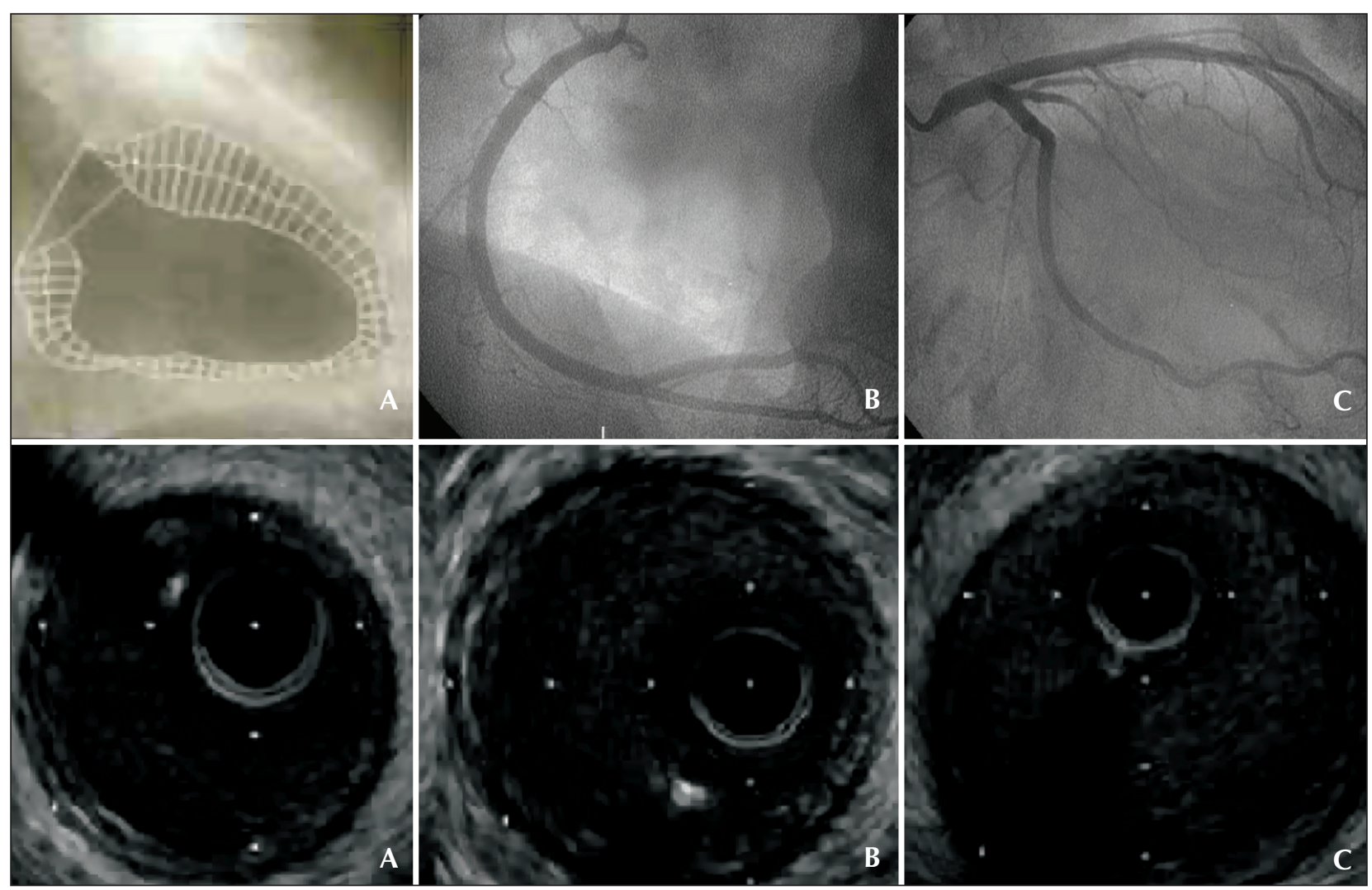

Figura 2 - Painel superior: Em A, ventriculografia em posição oblíqua anterior esquerda (OAE), demonstrando hipocinesia de parede inferior; em B e C, artérias coronárias direita e esquerda em oblíqua anterior esquerda cranial e posição oblíqua anterior direita convencional. Painel inferior: Ultrassonografia intracoronária da artéria coronária direita sem evidência de doença aterosclerótica ou obstrução $\left(\mathrm{A}^{\prime}=\right.$ distal; $\mathrm{B}^{\prime}=$ terço médio; $C^{\prime}=$ proximal).

que esses autores só tenham usado a angiografia como parâmetro de análise.

Caracteristicamente, os pacientes com infarto agudo do miocárdio com coronárias normais são mais jovens, com média de 40 anos, com maior incidência do sexo feminino e relato frequente de tabagismo ${ }^{9}$.

Dentre os mecanismos propostos para infarto agudo do miocárdio com coronárias normais estão: vasoespasmo coronário, trombólise in situ ou embolização com lise espontânea, uso de cocaína, miocardite viral, dissecção coronária, estado de hipercoagulabilidade, vasculite autoimune e intoxicação por monóxido de carbono $^{5}$. Dentre esses mecanismos, o espasmo é responsável por $15,5 \%$ dos $\operatorname{casos}^{7}$, geralmente em decorrência de disfunção endotelial sobre uma placa de aterosclerose, com liberação de fatores vasoconstritores e de menor produção de óxido nítrico ${ }^{9}$. Nesse aspecto, o tabagismo tem papel importante na gênese dessa anormalidade endotelial. A embolização responde por $2,2 \%$ dos $\operatorname{casos}^{7}$; caracteristicamente, a artéria descendente anterior é a mais acometida, e os pacientes podem ter histórico de doença valvar, endocardite e/ou trombo intracavitário ${ }^{5}$. A dissecção de artéria coronária é um evento muito raro ${ }^{5}$, decorrente da formação de hematoma entre as camadas média e adventícia, e em geral acomete mais mulheres (na proporção de 2:1), jovens, grávidas ou no puerpério, entidade em geral difícil de ser diagnosticada na angiografia, porém facilmente identificável à ultrassonografia intracoronária. No caso relatado, a ultrassonografia intracoronária foi importante, principalmente em afastar possíveis diagnósticos. No entanto, não esclareceu o provável mecanismo fisiopatológico, fato concordante com Kardasz e De Caterina ${ }^{7}$, que observaram que cerca de $66,2 \%$ dos casos de infarto agudo do miocárdio com coronárias normais ficam sem causa reconhecida.

\section{CONFLITO DE INTERESSES}

Os autores declararam inexistência de conflito de interesses.

\section{REFERÊNCIAS BIBLIOGRÁFICAS}

1. Thygesen K, Alpert JS, White HD; Joint ESC/ACCF/AHA/ WHF Task Force for the Redefinition of Myocardial Infarction. Universal definition of myocardial infarction. J Am Coll Cardiol. $2007 ; 50(22): 2173-95$. 
2. Moffa PJ. O eletrocardiograma e vetocardiograma no infarto miocárdio: diagnóstico topográfico, evolutivo e diferencial. In: Moffa PJ, Sanches PCR, editores. Tranchesi eletrocardiograma normal e patológico. São Paulo: Roca; 2001.

3. Atar S, Barbagelata A, Birnbaum Y. Electrocardiographic markers of reperfusion in ST-elevation myocardial infarction. Cardiol Clin. 2006;24(3):367-76.

4. Meisel SR, Dagan Y, Blondheim DS, Dacca S, Shochat M, Kazatsker M, et al. Transient ST-elevation myocardial infarction: clinical course with intensive medical therapy and early invasive approach, and comparison with persistent ST-elevation myocardial infarction. Am Heart J. 2008;155(5): 848-54.

5. Chandrasekaran B, Kurbaan AS. Myocardial infarction with angiographically normal coronary arteries. J R Soc Med. 2002;95(8):398-400.
6. Waller B. Nonatherosclerotic coronary heart disease. In: Fuster $\mathrm{V}$, Alexander RW, O'Rourke RA, editores. Hurst's the heart. 10 $0^{\text {th }}$ ed. Philadelphia: McGraw Hill; 2001. p.1168-70.

7. Kardasz I, De Caterina R. Myocardial infarction with normal coronary arteries: a conundrum with multiple aetiologies and variable prognosis: an update. J Intern Med. 2007;261(4): 330-48.

8. Larsen AI, Galbraith PD, Ghali WA, Norris CM, Graham MM, Knudtson ML; APPROACH Investigators. Characteristics and outcome of patients with acute myocardial infarction and angiographically normal coronary arteries. Am J Cardiol. 2005;95(2):261-3.

9. Sztajzel J, Mach F, Righetti A. Role of the vascular endothelium in patients with angina pectoris or acute myocardial infarction with normal coronary arteries. Postgrad Med J. 2000;76(891): 16-21. 